\title{
APPLICATION OF INTERVENTIONAL ULTRASOUND IN EMERGENCY MEDICINE CONDITIONS
}

\author{
Maciej Dudek ${ }^{1}$, Lukasz Szarpak ${ }^{1}$, Kurt Ruetzler ${ }^{2}$ \\ ${ }^{1}$ Lazarski University, Warsaw, Poland \\ ${ }^{2}$ Departments of Outcomes Research and General Anesthesiology, Anesthesiology Institute, Cleveland Clinic, Cleveland, Ohio, United States of America
}

\begin{abstract}
ULTRASONOGRAPHY is a modern diagnostic tool both in intensive care and emergency medicine. Small, portable and simple ultrasound devices have been introduced due to technological advances. The image quality, size, and weight of portable ultrasound devices are improving. Prehospital point-of-care ultrasonography may have an impact on the decision making in prioritizing initial treatment. First aid at the scene of the accident and transporting the patient to the hospital is a key element, which in the case of appropriate diagnostics allows you to fight life-threatening injuries. The intention of using ultrasound protocols is to shorten and simplify the ultrasound examination allowing to eliminate or find complications of an injury as soon as possible. The protocols used include elements of ultrasonography and echocardiography of the lung tissue, abdominal cavity, pelvis, large vessels or the eyeball. The intention of the authors of the article was to present to the reader the basic ultrasound protocols applicable to patients in emergency situations.
\end{abstract}

KEY WORDS: point-of-care ultrasonography, emergency medicine, ultrasonography, diagnostic imaging, review

Disaster Emerg Med J 2018; 3(4): 137-147

\section{INTRODUCTION}

Ultrasonography gives us the opportunity to examine the patient without prior preparation and without any implications of ultrasound [1-3]. It is the fastest and one of the most accurate diagnostic methods. Such examination can be carried out anywhere: street, ambulance, victim's house or the emergency department.

Point of Care $(P O C)$ ultrasound is a fairly young field of ultrasound, but the usefulness of protocols and research in this area is invaluable. In particular, when we are dealing with an unconscious patient and gathering an interview is impossible. Interventional ultrasound and Emergency Ultrasonography is not only for diagnostic purposes. It also allows you to monitor the condition of the victim by performing tests such as checking the optic nerve, giving us information on intracranial pressure $[4,5]$.

The assumption of POC ultrasound is to perform tests by medical personnel in order to assess the current state of the patient and, based on the result of this examination, undertake medical activities. We must distinguish these procedures from ordinary ultrasound examinations. In these studies, we do not assess the condition of organs, we only check the absence or presence of pathology that affects the functioning of the body. We have to treat the ultrasound like a "Stethoscope" - the first contact diagnostic tool. And as you know, the stethoscope serves all medical staff. 


\section{FOCUSED ASSESSMENT WITH SONOGRAPHY FOR TRAUMA PROTOCOL (FAST)}

The purpose of the procedure is to detect free fluid (blood) in the peritoneal cavity and pericardium. When the patient is showing hemodynamic instability with signs of anemization, we can assume that internal hemorrhage has occurred [6]. The fastest way to confirm this diagnosis is to perform the FAST procedure, i.e., Focused Assessment with Sonography for Trauma. Thanks to this examination, the patient can be quickly diagnosed and transported directly from the ambulance or ED to the operating theater, where they will be immediately operated, without unnecessary waste of time, which is critical for the patient's health and life. Examination FAST (extended) is an extended examination of the chest area and is intended to check if there is fluid (blood) or pneumothorax in the chest $[7,8]$.

Detection of free fluid in the abdominal cavity in patients after injuries using ultrasound is about $75 \%$ (up to $95 \%$ ). The specificity of the method is estimated at $98 \%$. While a physical examination is only $50-60 \%$ sensitive.

In the course of the study, it should be remembered that the ultrasound examination in a patient after an injury is not used to assess the extent of organ trauma. The information from the test is binary 0 - no liquid/air or 1 - liquid/air.

When performing the test, we usually use a Convex type head, i.e., a curved head in Poland commonly referred to as the abdominal head. In a situation where we do not have such a transducer, we can use a Phased Array type head, which is a head defined in the sector or cardiology sector in Poland (Fig. 1).

\section{Projections in the FAST study}

The whole procedure consists of 4 positions and 5 head applications within the abdominal cavity, chest, and pelvis (Fig. 2). To perform the test, we can use a Convex head or Phased Array head, i.e. a cardiac head with setting for abdominal applications [9]. There are also devices that have dedicated settings for POC procedures. The patient lies supine on a flat surface so that the fluid (blood) flows gravitationally into the open spaces.

The procedure begins with a sub-bridge projection in which we assess the heart and the pericardium. We start from this position because if we do not observe the movement of the heart muscle, it does not make sense to continue the procedure.

\section{Subcostal projection}

The position of the head and the space under examination: under the alveolar process in the transverse plane with the head marker pointing to the right (right side of the patient). The head is at a large angle and is directed to the left side of the chest (Fig. 3). We increase the depth of penetration of the device so that the image shows the whole heart and part of the liver, diaphragm, and lungs. When this projection is obtained, we must use adequate force for pressure to get a picture of the heart from under the surface of the ribs. In the absence of the desired image, we perform delicate fan-shaped movements, and left-right movements [10]. The obtained image should show the heart, part of the liver, and lungs. During image analysis, we direct our attention to the way the heart works, its regularity of work, and the quality of the ventricles and atrial contraction. We do not try to assess heart rate under
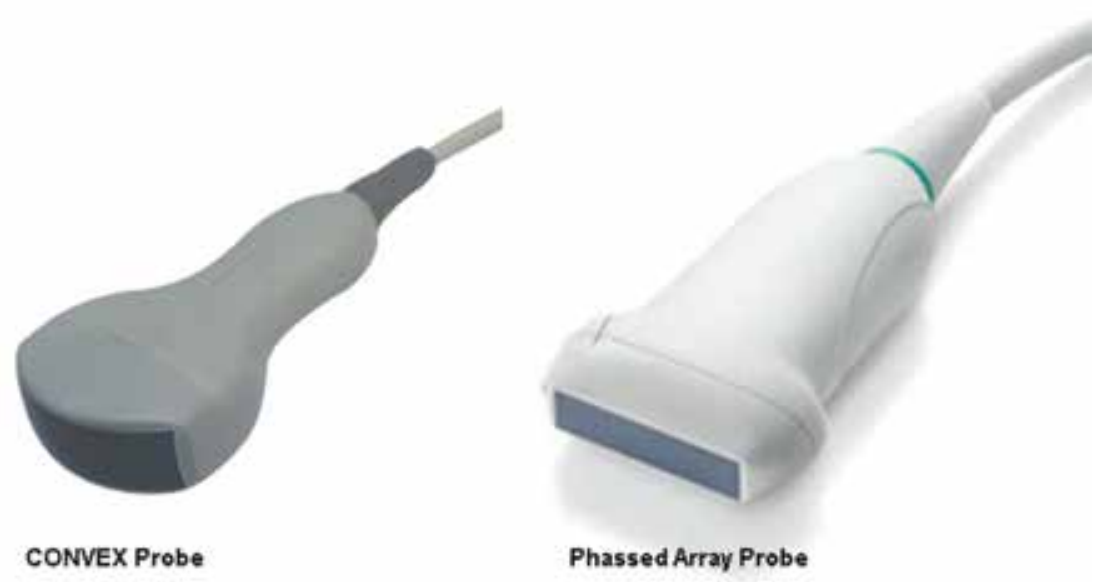

FIGURE 1. Differend ultrasononogrphy probes: (A) CONVEX probe; (B) Phassed Array probe 


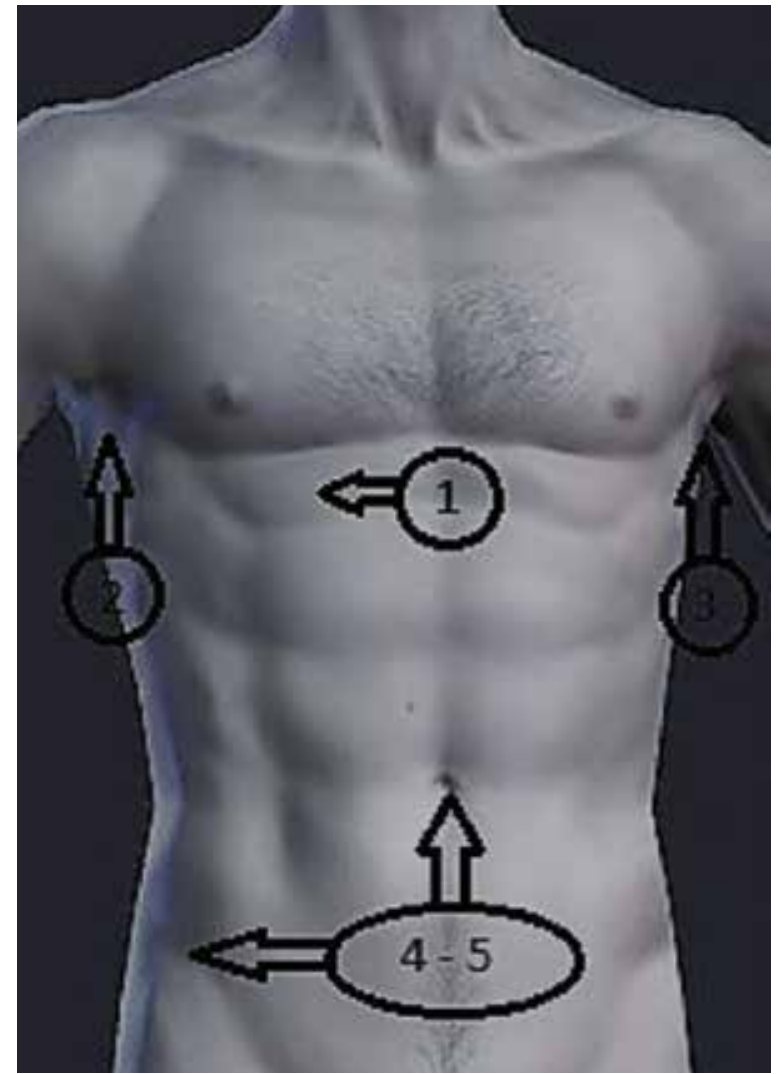

FIGURE 2. Ultrasound projections during FAST protocol

the account of flow speed or quality of valve work. However, irregular or non-synchronized heart work can be an indicator of the appearance of the pericardial sac. Blood in the ultrasound image is presented in black, just like any liquid.

If you cannot get the heart image from the postbridge position because of the patient's obesity or extensive trauma, we move the head to the chest and place it in the left parasternal longitudinal position. In this position, we keep the heads in a position perpendicular to the surface of the skin with a mark pointing in the cephalic direction.

\section{Right Upper Quadrant projection (RUQ)}

The position of the head and the area under study: periosteal area and hepatorenal space (Morison's pouch). We try to show the whole kidney, part of the diaphragm and the right lobe of the liver [11]. We arrange the head in the right axillary line of the anterior or medial plane in the sagittal and sagittal plane of the intercostal space from 7 to 9 . Head marker must be directed in the cephalothorax. This part of the procedure is imperative because by correctly orienting the head we can precisely determine the position of free fluid (Fig. 4).

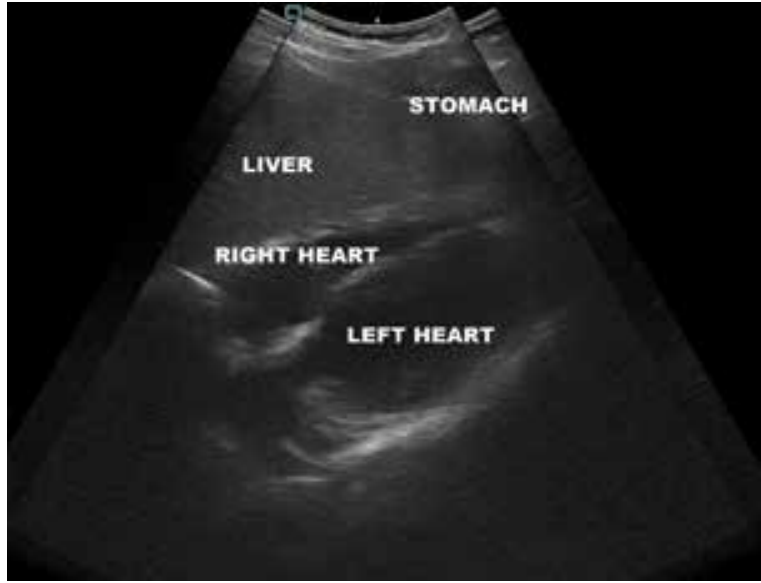

FIGURE 3. Ultrasound subcostal projection

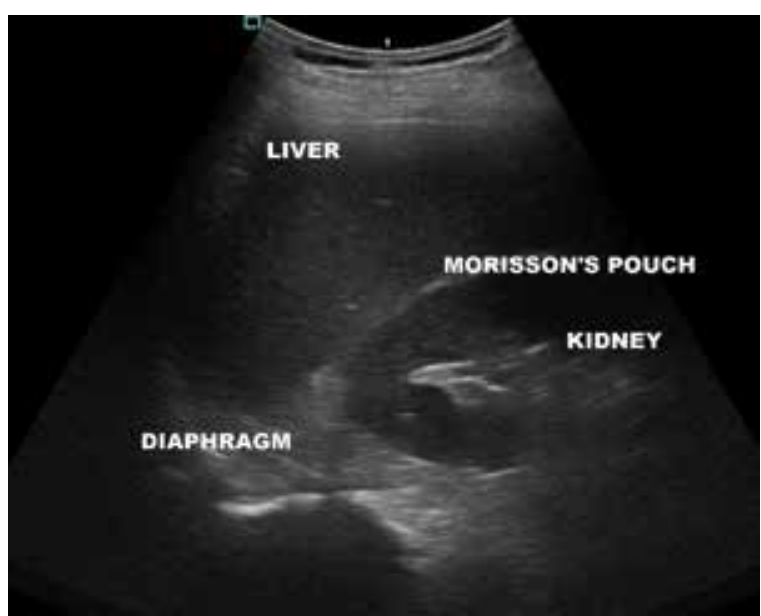

FIGURE 4. Ultrasound right upper quadrant projection

The intercostal spaces are not the same in different people, so often the image is significantly obscured by the acoustic shadow of the ribs. To bypass this problem, we must arrange the head along them. The ultrasound beam is very narrow because it is about $1.5 \mathrm{~mm}$, so imaging through the intercostal spaces should be a solution to this problem.

\subsection{Left Upper Quadrant projection (LUQ)}

The position of the head and the space under investigation: the peri-implant space (spleen-renal cantilever). In this projection, we try to present the entire left kidney, spleen, and part of the diaphragm [12]. We arrange the head in the left posterior axillary or axillary line in intercostal spaces from about ribs 5 to 7 in the sagittal or oblique plane with the head marker directed in the cephalothorax. We are proceeding here quite analogously to the RUQ 
projection, also important here to check the entire peripheral area (Fig. 5). In order to do this accurately, we have to move the head in the direction to and from the center of the axillary line in the middle and posterior axillary line. Free liquid blood will be manifested on the screen of the ultrasound as black. If the patient is conscious and it is known that his lungs are not damaged (we do eFAST or BLUE protocol), we can ask him to take a deep breath, which will greatly facilitate the full depiction of this space.

\section{Pelvis projection}

Space under study: lower abdominal cavity, and smaller pelvis. The test is much easier to do when the patient's bladder is full. For this reason, we should do this procedure before assembling the catheter, you can also clamp the catheter and wait for the bladder to fill or fill it with saline [13]. As we wrote in the description of the projection, it consists

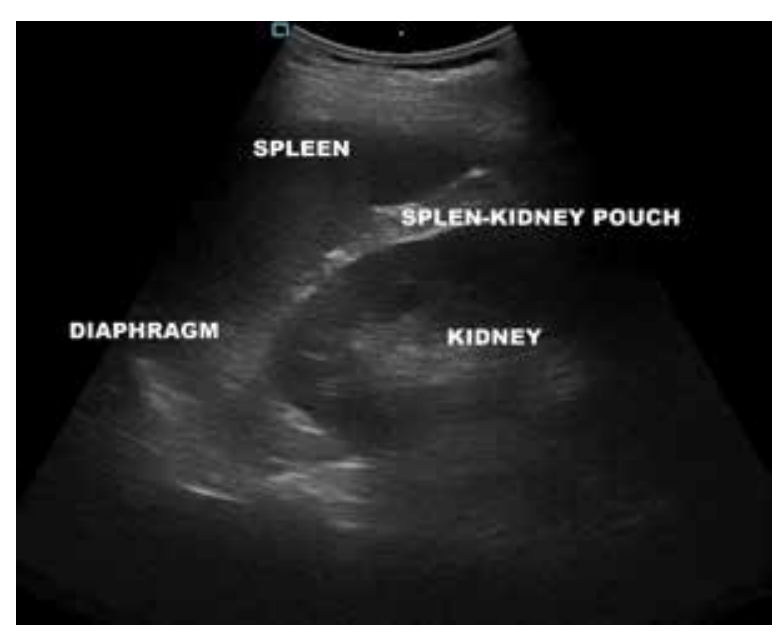

FIGURE 5. Ultrasound left upper quadrant projection

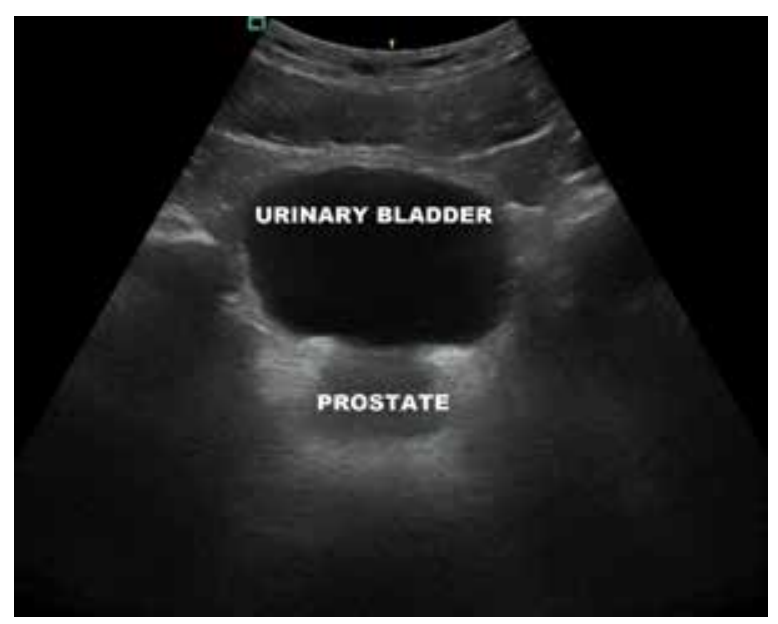

FIGURE 6. Ultrasound pelvis projection — Transverse position of two touchdowns, it does not matter much from which we start to remember both.

a) Head position: Transverse position - arrange the head above the pubic symphysis with the head marker pointing to the right (right side of the patient), heads lean towards the pelvis. In the picture, we try to visualize the bladder in the transverse plane of the Douglas pouch and pelvic organs (Fig. 6).

b) Head position: Arrow position - position the head above the pubic symphysis with the mark of the head directed in the sagittal plane in the sagittal plane. When moving from the transverse to the sagittal position, we rotate the head $90^{\circ}$, during rotation of the head we should keep the image of the bladder in the field of view (Fig. 7).

\section{EFAST PROTOCOL}

The eFAST (extended) study is an extended version of the FAST study [14]. To perform the eFAST test, add 3 positions of the head to the basic examination (Fig. 8). These items can be added anywhere in the study, but people with more experience recommend a method with the order of 1-6-2-34-5-7-8. It is logical because thanks to the similar positions of the head positioning, we can speed up the examination [15]. In the course of watching the lungs, we focus on two important phenomena, on the symptom of "sliding", or slipping the pleura, and viewing artifacts, or artificial creatures of the ultrasound device (Fig. 9). Physiologically, the lungs are filled with air, so we cannot visualize their flesh. Instead, we observe lines type A, B, C / Consolidation. The heads are placed in the left-collar, clavicular left and right positions 7-8 in 3-4th intercostal

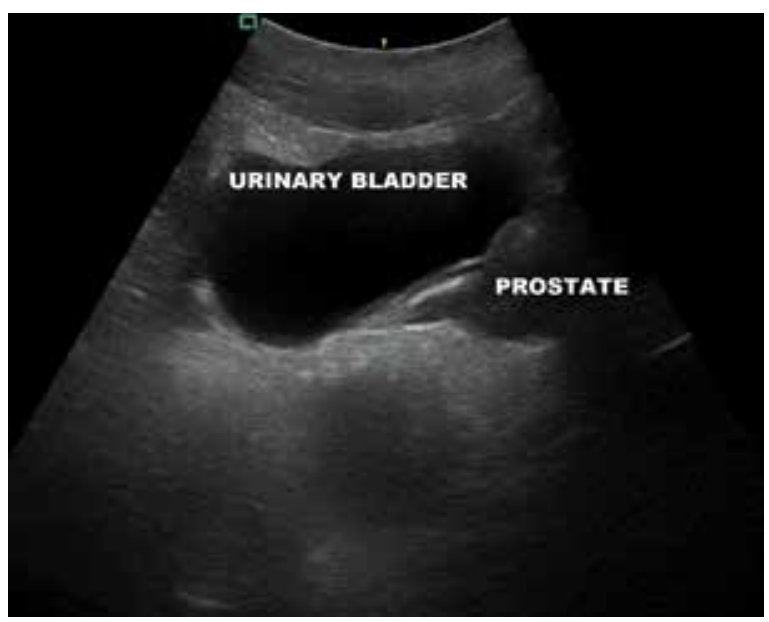

FIGURE 7. Ultrasound pelvis projection - Arrow position 


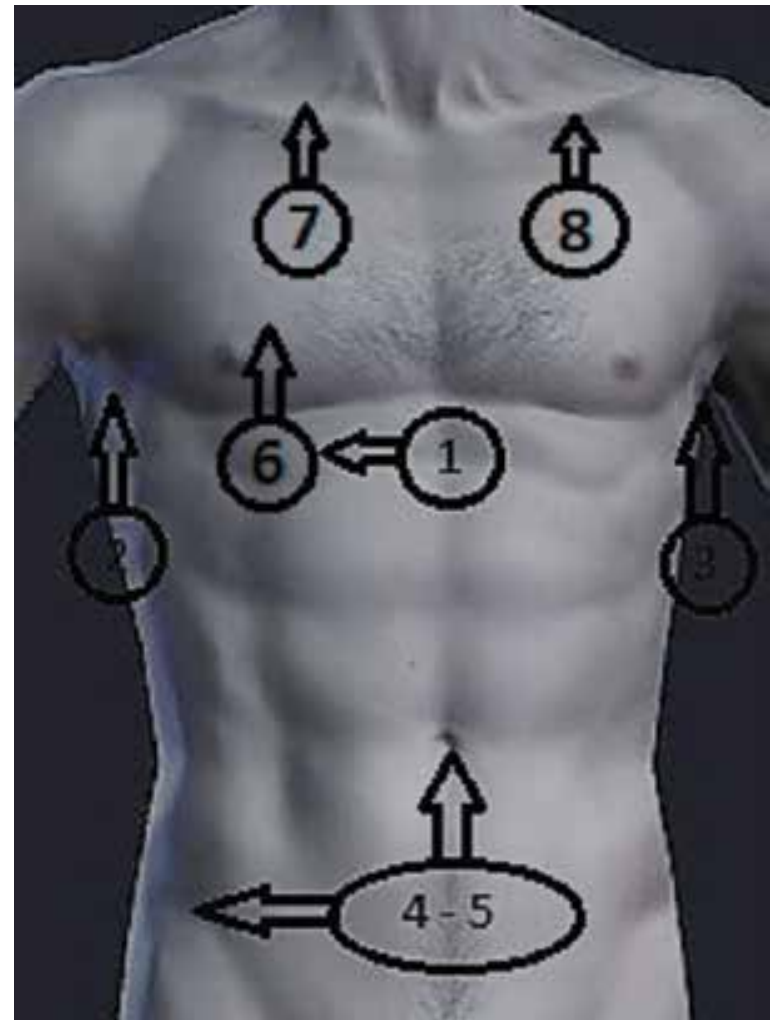

FIGURE 8. Ultrasound projections during eFAST protocol

and positions 6 in $8-9$. Pictures are not able to present the full study. In the pictures above, only the starting positions are presented, as well as the structures and organs that we should show [16]. We remember that the head presents a two-dimensional image, and the test space is three-dimensional, which means that we have to move the head in specific directions to get full information.

\section{BLUE PROTOCOL}

The BLUE (Bedside Lung Ultrasound in Emergency) procedure is used to diagnose the acute respiratory failure, which is a life-threatening condition $[17,18]$. This procedure can be performed both at the patient's home and in the hospital emergency department. The information we receive relates to the lungs and their condition: pneumothorax, pulmonary edema, or pulmonary embolism. The whole procedure should take up to three minutes. The test can be performed using any head, i.e., Phased Array, Convex, MicroConvex or Linear [19]. The recommended head for this procedure is the Microconvex or Phased Array. In the BLUE procedure, we view artifacts as in the case of e-FAST. At the same time, we should choose lung test applications (if we have

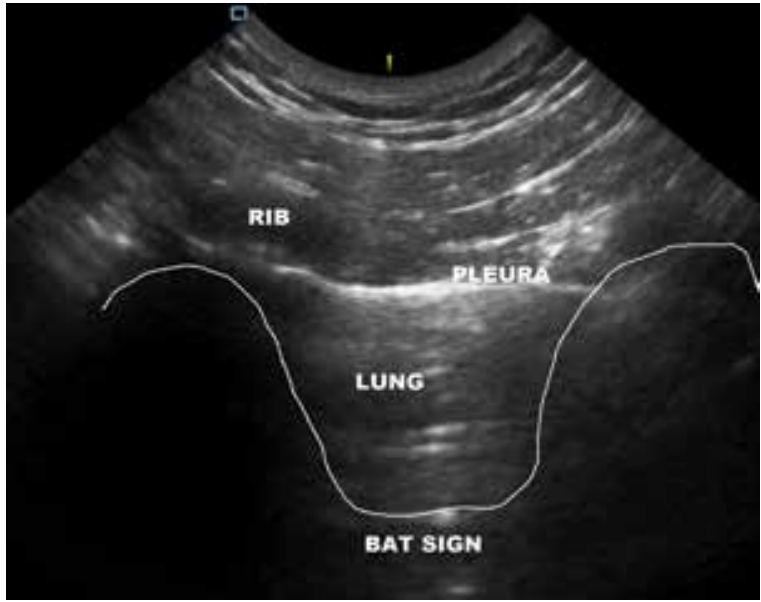

FIGURE 9. Ultrasound symptom of "sliding"

this option) or choose an abdominal application and turn off the options creating cross-linked beams on the device, because the device will create non-existent $B$ lines.

During the examination, the ultrasound machine can display several types of "lines" on the screen during the procedure:

- Lines A - These are normal artifacts (horizontal, parallel) resulting from the reflection of the pleural line in the air in the lungs, such lines will repeat at regular intervals - it is important that the distance between lines $A$ and the pleural line in the image must be constant otherwise, we may suspect Line $C$ or Consolidation.

- Lines B - These are abnormal (vertical, perpendicular) artifacts that arise from small quantities of liquid in the pleural space. Under normal conditions, the fluid is hypoechoic (dark, black) on the ultrasound image, but when it is surrounded by air, it gives a hyperechoic effect (light, white) through the effect of signal amplification in the fluid space. Remember that the $A$ lines do not coexist in the image with lines $B$.

- C-lines / Consolidations - These are lines created in a similar process to Line B, but in this case, the amount of fluid in the pleura is much greater. At the same time, the images appearing on the screen of the device do not have to come from the pleural space, but from the lungs themselves (Consolidations). We can observe such a picture when the lung parenchyma becomes visible during the examination.

The next and very important symptom observed on the screen during the lung examination is the "sliding symptom". It consists of checking the oc- 
currence of pleural "slipping" movements [20]. We observe the presence of a symptom in each of the touchdowns, this is the correct symptom. Sliding we can observe both in the presentation $B$ - Brightness and the presentation of $\mathrm{M}$ - Motion. In the B presentation, we obtain a position called "Bat Sign", which shows the image of the correct lung, and two adjacent intrapleural and shadows that they form. In this position, we also see visible pleural movement.

In the M presentation, we observe the "See Shore" Symptom - so-called the symptom of the beach, which similarly shows the correct image of the lungs, through the parallel arrangement of the lines that are the reflection of the pleura.

The test consists of three appendages on each side of the chest, the upper front point, the lower front point, and the PLAPS:

- The first point (front top) is between the third and fourth finger of the upper hand at their base

- The second front lower point is located at the height of the lower hand

- PLAPS point "posterior and/or lateral alveolar and/or pleural syndrome" - an intersection of the posterior axillary line and diaphragmatic/medial transverse line [21].

\section{FATE PROTOCOL}

The FATE (Focus Assessment Transthoracic in Emergency) protocol was created for the rapid assessment of hemodynamic disorders. It consists in the implementation of 4 touchdowns, of which 3 are closely related to cardiac imaging, sub-vertebral, apical and parasternal projection, and pleural projections in the middle axillary lines on both sides of the chest. This study will allow us to assess the condition of the pericardium: the presence of exudate, cardiac tamponade, cardiac assessment in patients with cardiac arrest, and the evaluation of the left ventricle systolic function $[22,23]$.

As in previous procedures, its execution time should not exceed 3 minutes. We do not have the ease of using any head for this protocol, we should use a Phased Array head because our projections are strictly echocardiographic [24]. As a last resort, we may try to use a Microconvex type head, but getting the correct image will be much more difficult.

- Substernal projection - this position offers excellent access to the heart in patients lying on the back. The head should be placed almost par- allel to the patient's skin just under the sternum towards the heart. From this position, we have a four-chamber view. By turning the head, you can get a short axis view. This is an important projection for monitoring pericardial effusions.

- The apical projection - the transducer is placed over the apex of the heart and directed parallel to the long axis of the heart, we get a four-chamber apical view. If we have a problem with the placement of the head, we can use the palpation technique to locate the apex of the heart (palpable heartbeats in the intercostal space). From the four-chamber view, we go to the two-chamber view. For this purpose, the transducer is rotated counter-clockwise. Two-cavity projection is used to assess the left and front walls of the left ventricle. Further rotation of the head gives the view of the long axis.

- Parasternal projection - the head is placed paradigmatic on the line connecting the apex of the heart with the center of the right clavicle at the left edge of the sternum. From this position, we get a black/five-pointed view. By turning the head (directing the marker to the right arm) we obtain a short axis. Image quality can be improved by turning the patient to the left side. A parasternal position is often called classic because in it we check the basic dimensions of the heart.

- Projection of the pleura - this projection is performed in the middle axillary lines. The head marker is directed to the patient's head. In this projection, we use the same principles that we apply to the BLUE procedure [25].

\section{RUSH PROTOCOL}

The RUSH protocol (Rapid Ultrasound in Shock and Hypotension) was created in 2009 by Dr. Weingart. The protocol is intended for the assessment of patients in shock and hypovolemia. The ultrasound schema was based on the HI-MAP abbreviation, according to which the projection sequence is performed. For this test, we use Phased Array or Convex head, as well as abdominal or FAST applications [26, 27]. In RUSH protocol we distinguish the following projecttions:

- H - Heart (heart),

- I - Inferior vena cava (main lower vein),

- M-Morison's pouch,

- A - Aorta,

- P-Pneumothorax (pneumothorax). 
The protocol starts with a heart projection $(1,2)$ (H-Heart), we obtain parasternal and apical projections to assess the pericardial sac (fluid presence) and the function of both chambers. Next, we present the lower main vein (3) (I-inferior vena cava) in the substernal space with the head marker directed to the patient's head. The next projection $(4,5)$ (M-Morison's Instruction) in this case means that we are looking at the spaces on both sides of the patient, both Morison's pouch and the kidney-spleen resection. While watching these spaces, we can move the head slightly in a cephalic way to visualize the basis of the lungs. The next projection $(6,7)$ (A-Aorta) is a combination of two projections. We start at the bladder with the marker pointing to the left and see the pelvic space to exclude free fluid in the peritoneal cavity, and then move towards the patient's head, finding the aorta and assessing its condition up to the sternum. The last protocol point $(8,9)$ (P-Pneumothorax) involves lung imaging to rule out pneumothorax, items as in the e-FAST study.

\section{THE PROCEDURE FOR MEASURING THE OPTIC NERVE DIAMETER}

The optic nerve is relatively easy to assess for the part of the nervous system that connects directly to the brain $[28,29]$. The optic nerve is the second cranial nerve, it means it is surrounded by the same tires as the brain [30]. When the intracranial pressure increases, the cerebrospinal fluid flows into the subarachnoid space, causing the pressure of the optic nerve to increase (in diameter). Knowing this relationship, we can indirectly determine intracranial pressure (ICP).

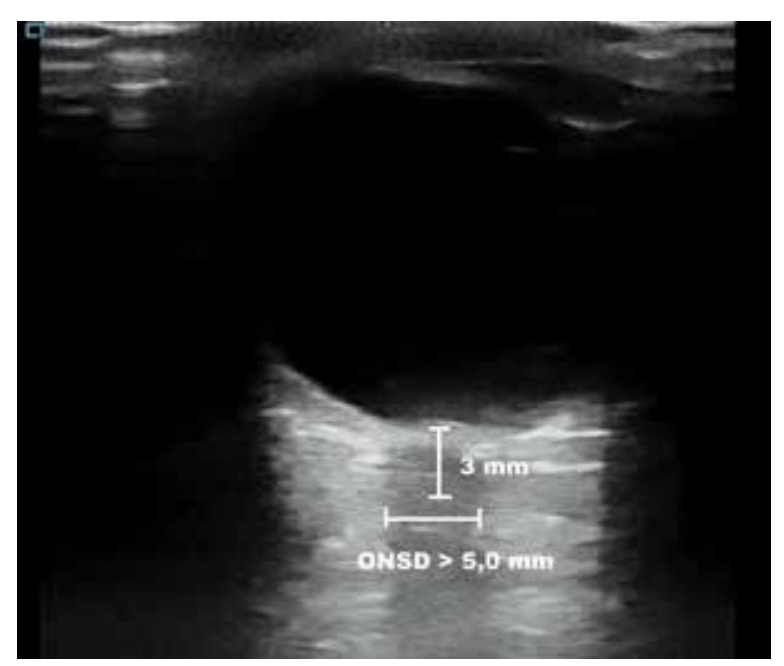

FIGURE 10. Optic nerve sheath diameter
The measurement is carried out using an 8-11 MHz high-frequency linear head [31, 32]. The structure of the eye is well-translucent for ultrasounds, so the test can be done with great accuracy every time. We perform the measurement when the patient has closed eyelids. The test time should not exceed one minute.

We measure the diameter of the optic nerve $3 \mathrm{~mm}$ behind the eyeball (Fig. 10). If we have a problem with finding a nerve, we can use a colored Doppler [33]. Anatomically, the artery and vein of the retina run inside the optic nerve, information marked with color will allow us to precisely determine the position of the nerve. The correct diameter of the optic nerve for an adult does not exceed $5 \mathrm{~mm}$, for a child $4.5 \mathrm{~mm}$, and for an infant $4 \mathrm{~mm}$.

\section{ASSESSMENT OF IRRIGATION DEGREE - IVC/AO}

A simple ultrasound procedure can also be used to assess the degree of filling of a vascular bed [34]. To determine its degree, we use the upper main vein, and more specifically its diameter (IVC; Fig. 11), which we compare with the width of the abdominal aorta (Ao; Fig. 12). This ratio is called the IVC/Ao index $[35,36]$.

For this measurement, we use a Convex type head. We position it in an anterior sagittal and axillary position with a marker directed to the patient's head. As the first we measure IVC at the height of the intrahepatic section, then we measure the aorta about $10 \mathrm{~mm}$ above the visceral trunk.

After obtaining the results, we substitute them for a simple IVC/Ao pattern where the standard deviation is $S D=1.2+/-0.2$.

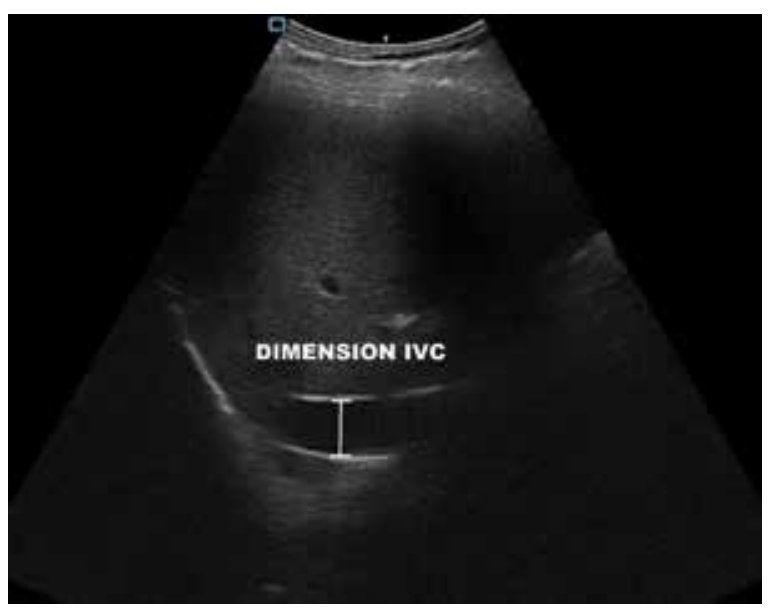

FIGURE 11. IVC width measurement 


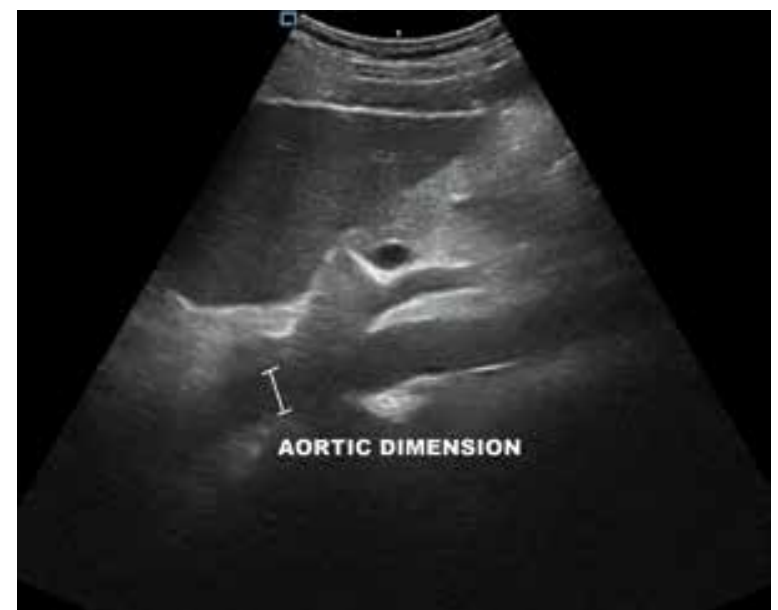

FIGURE 12. Aorta width measurement

\section{FEEL PROTOCOL}

The most important POC protocol dedicated to the management of cardiac arrest is FEEL (Focused Echocardiographic Evaluation in Life Support). This protocol was introduced by Breitkreutz in 2007 [37]. We perform this test using a Phased Array type head, and the procedure consists of 4 positions of the head: substernal, apical and parasternal in two axes [38, 39]. We must remember that each of the POC procedures is used only to detect an abnormal assessment of organs, therefore we only have 10 seconds to perform the assessment of the heart. Teamwork is very important during the conduct of the procedure.

This procedure consists of 10 points thanks to which the resuscitation team and the person performing ultrasound imaging will be able to use the most efficient time:

1) The team starts BLS and ALS resuscitation procedures - at least 5 full cycles.

2) The investigator informs the team that he starts the preparation of the ultrasound scanner.

3) Preparing the ultrasound for performing the procedure. We check whether the device is working properly, check whether the right head and application are selected. Also, if we have the appropriate depth of imaging, and whether the gel is on the head.

4) Adapting to the situation. The idea is to prepare yourself and the patient for the procedure. We set the device in the right place (to see the picture), if the patient has clothes, we take them off.

5) We tell the resuscitation team to start counting from 10 to 1 (in seconds - at the end of the cycle) and then began to measure the pulse.
6) We inform the team to interrupt the compressions after the end of the cycle to perform echocardiography. During the ultrasound procedure, the ventilation is maintained.

7) We apply the head to the patient in the substernal position while we are compressing and look for the right image with a fan-shaped motion. If this position is not possible to obtain (injury, obesity), we use intercostal spaces and parasternal positions in the long axis.

8) When the team is finished counting from 10 to 1 , the compression is interrupted, and the ultrasound scan begins. We have 3 seconds to find the heart and 3 seconds to evaluate it. If we do not find the heart, or we do not observe its movements, we inform the team about returning to full resuscitation for 5 subsequent cycles, after which we return with the head in the next position. Compressions cannot be interrupted for more than 10 seconds.

9) We inform the team about returning to resuscitation activities.

10) After the team returns to resuscitation, we inform the others about the conclusions we noticed during the test:

- Wall movement present / absent

- Pericardium tamponade

- Presence of PEA (pulseless electrical activity) / alleged PEA

- Poor image / no visibility

- etc.

This procedure gives us the opportunity to truly evaluate the performance of myocardium and the quality of its work and the effectiveness of resuscitation $[40,41]$.

\section{DEEP VEIN THROMBOSIS DVT}

We perform a test for the presence of deep vein thrombosis in a patient with swelling and/or pain in the calf area. A properly constructed vein collapses during compression, unlike arteries. When the venous vessel is destroyed, the walls become hardy and the functioning of the venous valves is impaired [42]. We will look for the first of these two symptoms during the procedure. The study is optimally performed using two heads: Convex type transducer for examination of iliac veins and Linear head for evaluation of the rest of the venous system of the lower limb. There are different methods of performing this test, but I think that the most accurate 


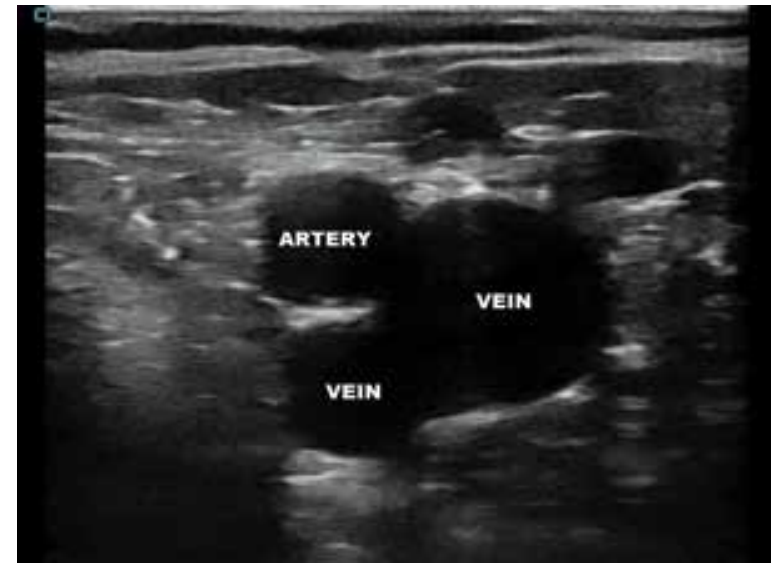

FIGURE 13. "Mickey Mouse" ultrasound image during deep vein thrombosis

and the fastest method is the 3-point compression method [43].

To perform this procedure, we lay the patient in a supine position and rotate the examined limb, about $10-15^{\circ}$ outside. For the assessment of the popliteal segment, we can put the patient on the side and bend his leg in the knee joint by $12-30^{\circ}$.

The heads are applied transversely to the course of the vessel with the marker pointing to the left, i.e. to the right side of the patient. During each position, we must compress the venous vessel and assess its susceptibility to compression. If necessary, we should document our activities.

The procedure starts with the groin in which we are looking for the so-called "Mickey Mouse" image, i.e. the saphenofemoral runoff (Fig. 13). This is the first position. Then we go along the femoral vein to the next section, where I repeat the procedure. Next, we move to the height of the popliteal vein and perform a pressure test [44]. The whole process is repeated on the other limb.

\section{SUMMARY}

As presented in the above article, interventional ultrasonography and emergency procedures can help diagnose and implement treatment in patients with many disease entities that we will not be able to recognize during an interview or physical examination $[45,46]$. The ultrasound machine gives us the possibility of a quick and non-invasive view of the problem directly, with the simultaneous possibility of indicating the cause of the ill condition of the patient without any doubts - especially in unconscious patients. The ultrasound machine is a small device that can be carried even in a pocket. Access to such devices for emergency medical services will not only increase the effectiveness in the diagnosis of injuries, but also enable continuous monitoring of the patient's condition, either in the ambulance or on the emergency department.

\section{REFERENCES:}

11. Whitson MR, Mayo PH. Ultrasonography in the emergency department. Crit Care. 2016; 20(1): 227, doi: 10.1186/s13054-016-1399-x, indexed in Pubmed: 27523885.

12. Marin J, Lewiss R. Point-of-Care Ultrasonography by Pediatric Emergency Medicine Physicians. Pediatric Emergency Care. 2015; 31(7): 525, doi: 10.1097/pec.0000000000000492.

13. Marin JR, Lewiss RE. American Academy of Pediatrics, Committee on Pediatric Emergency Medicine, Society for Academic Emergency Medicine, Academy of Emergency Ultrasound, American College of Emergency Physicians, Pediatric Emergency Medicine Committee, World Interactive Network Focused on Critical Ultrasound. Point-ofcare ultrasonography by pediatric emergency medicine physicians. Pediatrics. 2015; 135(4): e1113-e1122, doi: 10.1542/peds.20150343, indexed in Pubmed: 25825532.

14. Barbariol F, Vetrugno L, Pompei $L$, et al. Point-of-care ultrasound of the diaphragm in a liver transplant patient with acute respiratory failure. Crit Ultrasound J. 2015; 7: 3, doi: 10.1186/s13089-015-0021-9, indexed in Pubmed: 25859317.

15. Lui JK, Banauch GI. Diagnostic Bedside Ultrasonography for Acute Respiratory Failure and Severe Hypoxemia in the Medical Intensive Care Unit: Basics and Comprehensive Approaches. J Intensive Care Med. 2017; 32(6): 355-372, doi: 10.1177/0885066616658475, indexed in Pubmed: 27402396.

16. Richards JR, McGahan JP. Focused Assessment with Sonography in Trauma (FAST) in 2017: What Radiologists Can Learn. Radiology. 2017; 283(1): 30-48, doi: 10.1148/radiol.2017160107, indexed in Pubmed: 28318439.

17. Todsen T, Tolsgaard MG. Assessment of competence in focused assessment with sonography for trauma (FAST) examination. J Trauma Acute Care Surg. 2016; 80(2): 353, doi: 10.1097/TA.0000000000000920, indexed in Pubmed: 26595706.

18. Fornell Pérez R. Focused assessment with sonography for trauma (FAST) versus multidetector computed tomography in hemodynamically unstable emergency patients. Radiologia. 2017; 59(6): 531-534, doi: 10.1016/j.rx.2016.11.007, indexed in Pubmed: 28190526.

19. Calder BW, Vogel AM, Zhang J, et al. Focused assessment with sonography for trauma in children after blunt abdominal trauma: A multi-institutional analysis. J Trauma Acute Care Surg. 2017; 83(2): 218-224, doi: 10.1097/TA.0000000000001546, indexed in Pubmed: 28590347.

20. Chung GK, Gyllenhammer RG, Baker EL, et al. Effects of simulation-based practice on focused assessment with sonography for trauma (FAST) window identification, acquisition, and diagnosis. Mil Med. 
2013; 178(10 Suppl): 87-97, doi: 10.7205/MILMED-D-13-00208, indexed in Pubmed: 24084309.

21. Rozycki GS, Ochsner MG, Feliciano DV, et al. Early detection of hemoperitoneum by ultrasound examination of the right upper quadrant: a multicenter study. J Trauma. 1998; 45(5): 878-883, indexed in Pubmed: 9820696.

22. O'Brien KM, Stolz LA, Amini R, et al. Focused Assessment With Sonography for Trauma Examination: Reexamining the Importance of the Left Upper Quadrant View. J Ultrasound Med. 2015; 34(8): 1429-1434, doi: 10.7863/ultra.34.8.1429, indexed in Pubmed: 26206829.

23. Shaukat NM, Copeli N, Desai P. The Focused Assessment With Sonography For Trauma (FAST) Examination And Pelvic Trauma: Indications And Limitations. Emerg Med Pract. 2016; 18(3): 1-20, 24; quiz 20, indexed in Pubmed: 26881977.

24. Frink $M$, Lechler $P$, Debus $F$, et al. Multiple Trauma and Emergency Room Management. Dtsch Arztebl Int. 2017; 114(29-30): 497-503, doi: 10.3238/arztebl.2017.0497, indexed in Pubmed: 28818179.

25. Pencil K. eFAST Simulation Training for Trauma Providers. J Trauma Nurs. 2017; 24(6): 376-380, doi: 10.1097/JTN.0000000000000329, indexed in Pubmed: 29117056.

26. Montoya J, Stawicki SP, Evans DC, et al. From FAST to E-FAST: an overview of the evolution of ultrasound-based traumatic injury assessment. Eur J Trauma Emerg Surg. 2016; 42(2): 119-126, doi: 10.1007/ s00068-015-0512-1, indexed in Pubmed: 26038031.

27. Lichtenstein DA. BLUE-protocol and FALLS-protocol: two applications of lung ultrasound in the critically ill. Chest. 2015; 147(6): 1659-1670, doi: 10.1378/chest.14-1313, indexed in Pubmed: 26033127.

28. Lichtenstein DA, Mezière GA. Relevance of lung ultrasound in the diagnosis of acute respiratory failure: the BLUE protocol. Chest. 2008; 134(1): 117-125, doi: 10.1378/chest.07-2800, indexed in Pubmed: 18403664.

29. Seyedhosseini J, Bashizadeh-Fakhar G, Farzaneh S, et al. The impact of the BLUE protocol ultrasonography on the time taken to treat acute respiratory distress in the ED. Am J Emerg Med. 2017; 35(12): 1815-1818, doi: 10.1016/j.ajem.2017.06.007, indexed in Pubmed: 28624146.

30. Khosla R. Bedside lung ultrasound in emergency (BLUE) protocol: a suggestion to modify. Chest. 2010; 137(6): 1487, doi: 10.1378/ chest.09-3123, indexed in Pubmed: 20525667.

31. Lichtenstein D. Lung ultrasound in the critically ill. Curr Opin Crit Care. 2014; 20(3): 315-322, doi: 10.1097/MCC.0000000000000096, indexed in Pubmed: 24758984.

32. Nishigami K. Point-of-care echocardiography for aortic dissection, pulmonary embolism and acute coronary syndrome in patients with killer chest pain: EASY screening focused on the assessment of effusion, aorta, ventricular size and shape and ventricular asynergy. J Echocardiogr. 2015; 13(4): 141-144, doi: 10.1007/s12574-015-02651, indexed in Pubmed: 26497152.

33. Nazerian $P$, Vanni $S$, Castelli $M$, et al. Diagnostic performance of emergency transthoracic focus cardiac ultrasound in suspected acute type A aortic dissection. Intern Emerg Med. 2014; 9(6): 665-670, doi: 10.1007/s11739-014-1080-9, indexed in Pubmed: 24871637.
34. Rossinen J. FATE echocardiography at the emergency outpatient unit. Duodecim. 2016; 132(8): 783-790, indexed in Pubmed: 27244938.

35. Jensen MB, Sloth E. [Transthoracic ultrasound: a necessary standard within intensive, acute and pre-hospital medicine]. Ugeskr Laeger. 2006; 168(50): 4393-4396, indexed in Pubmed: 17217863.

36. Blanco P, Aguiar FM, Blaivas M. Rapid Ultrasound in Shock (RUSH) Velocity-Time Integral: A Proposal to Expand the RUSH Protocol. J Ultrasound Med. 2015; 34(9): 1691-1700, doi: 10.7863/ultra.15.14.08059, indexed in Pubmed: 26283755.

37. Estoos E, Nakitende D. Diagnostic Ultrasound Use In Undifferentiated Hypotension. StatPearls [Internet]. Treasure Island (FL): StatPearls Publishing. 2018 Jan-.2018 0ct 27.

38. Ladny M, Szarpak L, Smereka J, et al. A comparison of comfort assessment of NECKLITE vs. NeXsplint cervical collar. Pilot data. Am J Emerg Med. 2018; 36(11): 2127-2128, doi: 10.1016/j.ajem.2018.03.078, indexed in Pubmed: 29752163.

39. Steinborn $M$, Friedmann $M$, Hahn $H$, et al. Normal values for transbulbar sonography and magnetic resonance imaging of the optic nerve sheath diameter (ONSD) in children and adolescents. Ultraschall Med. 2015; 36(1): 54-58, doi: 10.1055/s-0034-1385012, indexed in Pubmed: 25140497.

40. Rajajee V, Vanaman M, Fletcher JJ, et al. Optic nerve ultrasound for the detection of raised intracranial pressure. Neurocrit Care. 2011; 15(3): 506-515, doi: 10.1007/s12028-011-9606-8, indexed in Pubmed: 21769456.

41. Hylkema C. Optic Nerve Sheath Diameter Ultrasound and the Diagnosis of Increased Intracranial Pressure. Crit Care Nurs Clin North Am. 2016; 28(1): 95-99, doi: 10.1016/j.cnc.2015.10.005, indexed in Pubmed: 26873762.

42. Raffiz M, Abdullah JM. Optic nerve sheath diameter measurement: a means of detecting raised ICP in adult traumatic and non-traumatic neurosurgical patients. Am J Emerg Med. 2017; 35(1): 150-153, doi: 10.1016/j.ajem.2016.09.044, indexed in Pubmed: 27852525.

43. Kavi T, Gupta A, Hunter K, et al. Optic Nerve Sheath Diameter Assessment in Patients with Intracranial Pressure Monitoring. Cureus. 2018; 10(11): e3546, doi: 10.7759/cureus.3546, indexed in Pubmed: 30648078 .

44. Vaish H, Kumar V, Anand R, et al. The Correlation Between Inferior Vena Cava Diameter Measured by Ultrasonography and Central Venous Pressure. Indian J Pediatr. 2017; 84(10): 757-762, doi: 10.1007/ s12098-017-2433-y, indexed in Pubmed: 28868586.

45. Varol A, Basa S, Topsakal A, et al. Assessment of synovial vascularization by power Doppler ultrasonography in TMJ internal derangements treated arthroscopically. Br J Oral Maxillofac Surg. 2008; 46(8): 625-630, doi: 10.1016/j.bjoms.2008.04.022, indexed in Pubmed: 18584928.

46. Koster $\mathrm{G}$, van der Horst ICC. Critical care ultrasonography in circulatory shock. Curr Opin Crit Care. 2017; 23(4): 326-333, doi: 10.1097/ MCC.0000000000000428, indexed in Pubmed: 28590257.

47. Bhanji F, Donoghue AJ, Wolff MS, et al. Part 14: Education: 2015 American Heart Association Guidelines Update for Cardiopulmonary 
Resuscitation and Emergency Cardiovascular Care. Circulation. 2015; 132(18 Suppl 2): S561-S573, doi: 10.1161/CIR.0000000000000268, indexed in Pubmed: 26473002.

48. Breitkreutz R, Walcher F, Seeger FH. Focused echocardiographic evaluation in resuscitation management: concept of an advanced life support-conformed algorithm. Crit Care Med. 2007; 35(5 Suppl): S150-S161, doi: 10.1097/01.CCM.0000260626.23848.FC, indexed in Pubmed: 17446774.

49. Price $S$, Uddin S, Quinn T. Echocardiography in cardiac arrest. Curr Opin Crit Care. 2010; 16(3): 211-215, doi: 10.1097/ MCC.0b013e3283399d4c, indexed in Pubmed: 20463464.

50. Breitkreutz R, Price S, Steiger HV, et al. Emergency Ultrasound Working Group of the Johann Wolfgang Goethe-University Hospital, Frankfurt am Main. Focused echocardiographic evaluation in life support and peri-resuscitation of emergency patients: a prospective trial. Resuscitation. 2010; 81(11): 1527-1533, doi: 10.1016/j.resuscitation.2010.07.013, indexed in Pubmed: 20801576.

51. Price $S$, Ilper $H$, Uddin $S$, et al. Peri-resuscitation echocardiography: training the novice practitioner. Resuscitation. 2010; 81(11): 1534-1539, doi: 10.1016/j.resuscitation.2010.07.001, indexed in Pubmed: 20727655.
52. Maufus M, Elias A, Barrellier MT, et al. French Society for Vascular Medicine. Diagnosis of deep vein thrombosis recurrence: Ultrasound criteria. Thromb Res. 2018; 161: 78-83, doi: 10.1016/j.thromres.2017.11.004, indexed in Pubmed: 29216479.

53. Pomero F, Dentali F, Borretta $V$, et al. Accuracy of emergency physician-performed ultrasonography in the diagnosis of deep-vein thrombosis: a systematic review and meta-analysis. Thromb Haemost. 2013; 109(1): 137-145, doi: 10.1160/TH12-07-0473, indexed in Pubmed: 23138420.

54. Valentin ML, Clemens R, Thalhammer C. Duplex ultrasound of deep vein thrombosis of the leg. Dtsch Med Wochenschr. 2016; 141(13): 946-949, doi: 10.1055/s-0042-108930, indexed in Pubmed: 27359313.

55. Matthew Fields J, Davis J, Alsup C, et al. Accuracy of Point-of-care Ultrasonography for Diagnosing Acute Appendicitis: A Systematic Review and Meta-analysis. Acad Emerg Med. 2017; 24(9): 1124-1136, doi: 10.1111/acem.13212, indexed in Pubmed: 28464459.

56. Liu RB, Donroe JH, McNamara RL, et al. The Practice and Implications of Finding Fluid During Point-of-Care Ultrasonography: A Review. JAMA Intern Med. 2017; 177(12): 1818-1825, doi: 10.1001/jamainternmed.2017.5048, indexed in Pubmed: 29059269. 